\title{
When a normal hearing test is just the beginning
}

Veronica Kennedy Claire Wilson Dafydd Stephens

J R Soc Med 2006;99:417-420

Where hearing difficulties exist despite a normal hearing test, consider the presence of an underlying subclinical disorder or a developing problem.

\section{CASE HISTORIES}

\section{Case 1}

A 50-year-old woman presented with a 3-month history of hearing difficulty in her left ear. This was related to a sudden feeling of blockage in her left ear with perceived distortion. The sensation of blockage cleared initially, but then recurred and persisted. Audiometrically her hearing was normal. An initial diagnosis of King-Kopetzky syndrome was made and further hearing tests were organized. Her transient-evoked otoacoustic emissions were poor. As the perception of sound can be affected by both peripheral and central auditory processing disorders, central speech tests were performed and found to be normal. Her hearing difficulty persisted, as did the sensation of blockage in her left ear. It was felt her symptoms were now suggestive of endolymphatic hydrops or a variant of Menière's disorder rather than King-Kopetzky syndrome. Her symptoms initially improved with diuretic use. A low salt diet had not been helpful. Nineteen months after her initial presentation her hearing had deteriorated. She now had a low frequency sensorineural loss (Figure 1) but with good speech recognition. A magnetic resonance imaging scan of her internal auditory meati and brain was normal. This evolving picture supported the diagnosis of Menière's disorder.

\section{Case 2}

A woman aged 53 reported a hearing loss in her left ear after an upper respiratory tract infection, although she had initially felt it recovered completely. She had no noted tinnitus or dizziness, nor any neurological problem. Audioscan audiometry and distortion product otoacoustic emissions were normal. She reattended 4 years later complaining of progressive hearing loss, tinnitus, numbness of the left side of her face and a mild disturbance of balance over the preceding year. She was noted to have reduced

Welsh Hearing Institute, University Hospital of Wales, Cardiff CF14 4XW, UK

Correspondence to: Dr Veronica Kennedy

E-mail: vkennedy@doctors.org.uk

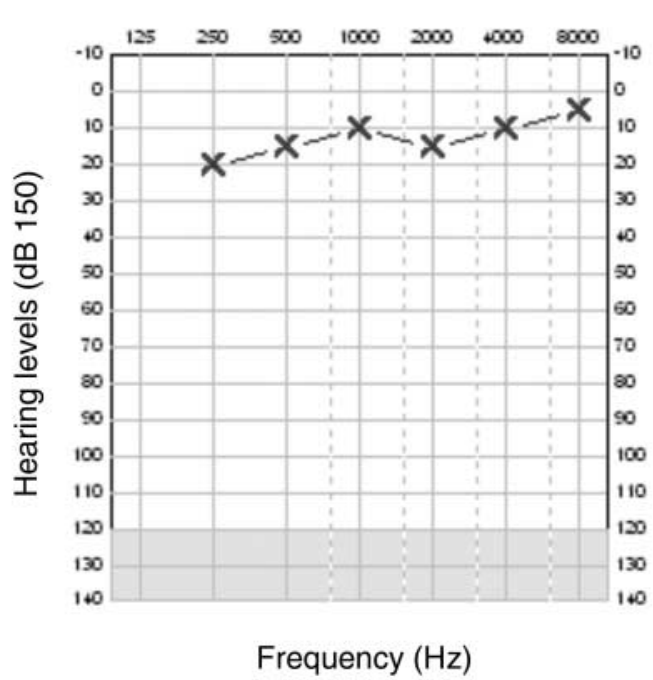

(a)

(b)

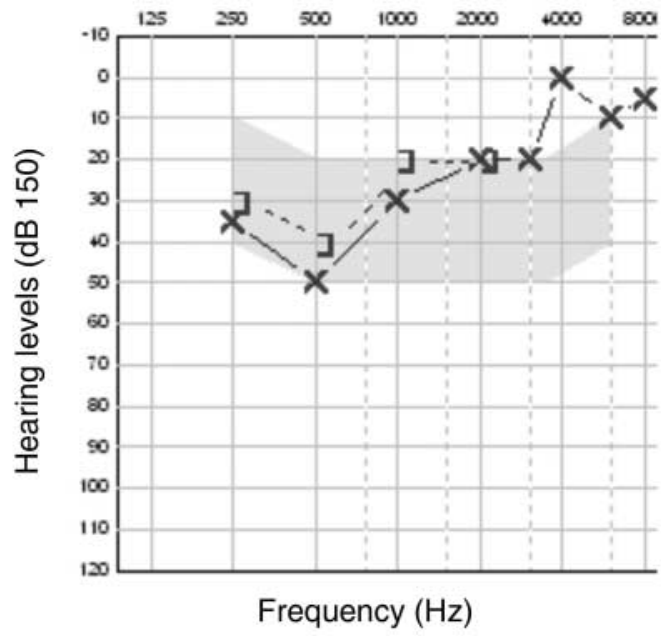

Figure 1 Pure tone audiogram (left ear): progressive sensorineural loss due to endolymphatic hydrops. (a) Pure tone audiogram: initial test; (b) pure tone audiogram: 19 months later $\mathrm{X}$, left masked air conduction; $\mathbf{I}$, left masked bone conduction

facial sensation in the area supplied by the mandibular branch of the left trigeminal nerve and a reduced left corneal reflex. Pure tone audiometry (Figure 2) showed a marked deterioration in her hearing thresholds. Magnetic resonance imaging (Figure 3) demonstrated a partly cystic, partly solid extra axial mass extending into a widened left internal auditory meatus and displacing the fifth cranial nerve at its root entry zone. Radiologically this was consistent with a large vestibular Schwannoma. Peroperatively this mass was found to be a facial neuroma. Postoperatively she had a persisting profound left hearing loss, a seventh lower motor nerve palsy and ataxia.

\section{Case 3}

A 23-year-old medical student on an ENT attachment reported having been aware of hearing difficulties since she 

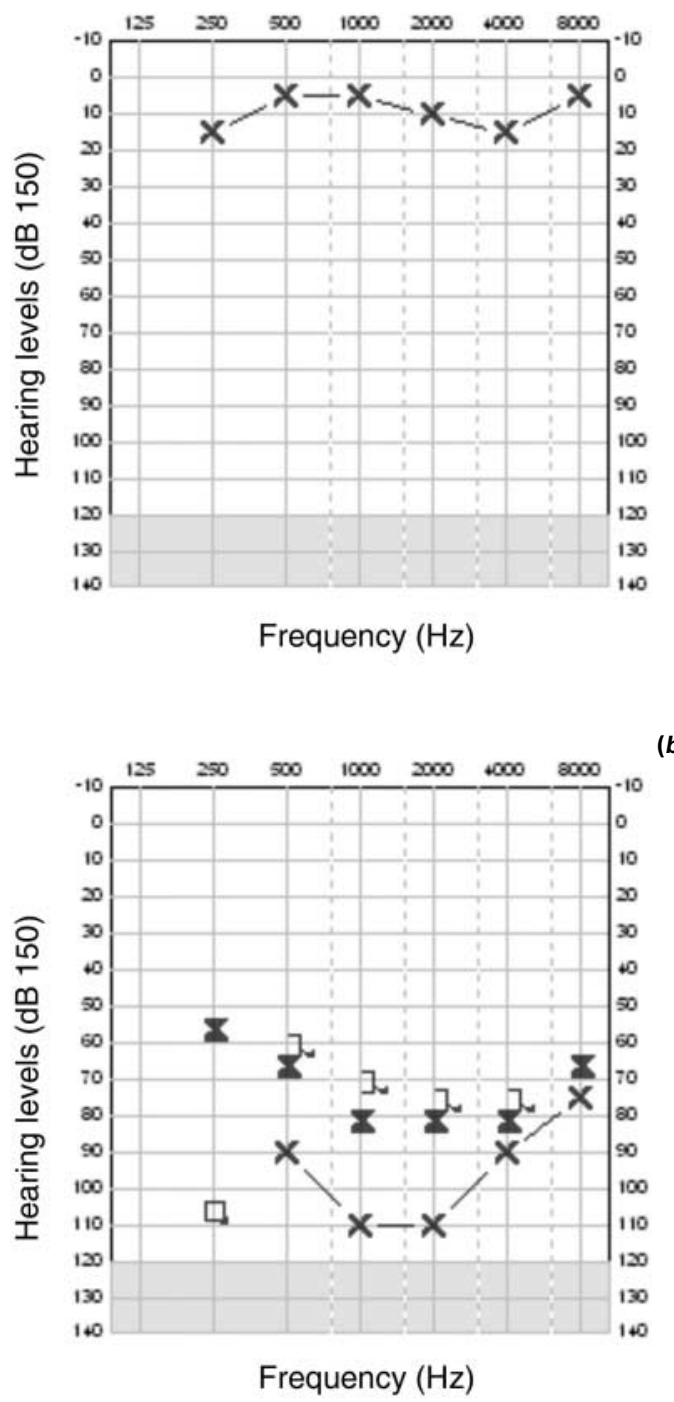

Figure 2 Pure tone audiometry (left ear): progressive sensorineural hearing loss due to facial neuroma. (a) Pure tone audiogram: initial test; (b) pure tone audiogram: 4 years later. $\mathrm{X}$, left masked air conduction; $\mathbf{X}$, unmasked air conduction; $\mathbf{I}$, left masked bone conduction

was 12 years old, which she felt was having a negative impact on her social life. A pure tone audiogram (Figure 4) was reported as being normal and she was subsequently referred to the audiological medicine department with a diagnosis of King-Kopetzky syndrome. On questioning, it was noted that both her father and elder brother had hearing problems. Békésy audiometry tracings showed a bilateral mild mid-frequency sensorineural hearing loss. On review of her initial pre tone audiogram a mild low frequecy loss was noted. Distortion product oto-acoustic emissions showed a mid-frequency dip. A similar pattern was noted on Békésy audiometry testing of both her father and brother (Figure 5) supporting a diagnosis of a nonsyndromal autosomal dominant hearing impairment. She

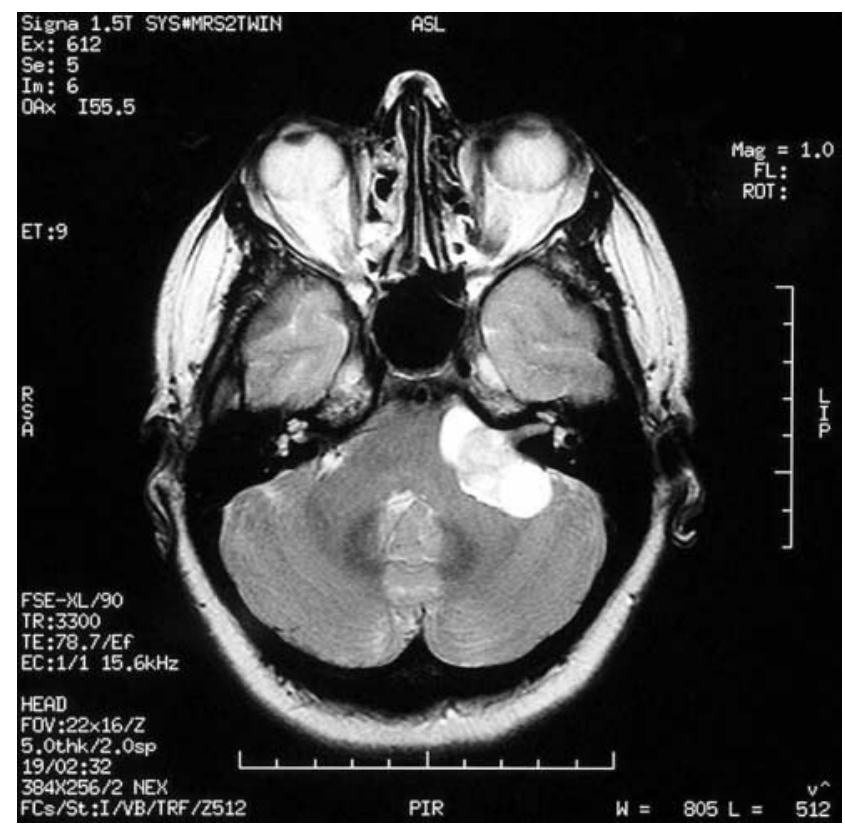

Figure 3 Magnetic resonance imaging scan

strategies and environmental aids including electronic stethoscopes.

\section{DISCUSSION}

Hearing difficulties in adults who have normal thresholds on pure tone audiometry are a common complaint, but also commonly ignored. Those that are not ignored often tend to be given a presumptive diagnosis of King-Kopetzky syndrome $^{1}$ or obscure auditory dysfunction ${ }^{2}$ without any provision being made for further investigation or monitoring. Both these terms describe the condition in which an individual complains of hearing difficulties, particularly in the presence of background noise, but has a normal pure tone audiogram and no obvious cause for the hearing difficulty. It may, in some cases, be a provisional diagnosis until a more substantive diagnosis is made. It is a relatively common condition with a prevalence of $5 \%-37 \%$ in patients complaining of hearing problems in an audiology clinic. ${ }^{3,4}$ It is also a heterogeneous condition. ${ }^{1,2}$ On investigation, causes may be identified which include a peripheral or central auditory dysfunction, psychological disorder or a combination of these factors. The awareness of a hearing difficulty may result in a stress response or be the initial presentation of an evolving pathology or late onset genetic hearing impairment.

The most commonly used test procedures are crude compared with the complex nature of the auditory system and provide only a narrow view of the hearing process. The most common hearing test used, pure tone audiometry, is generally used to test one or two frequencies within an 

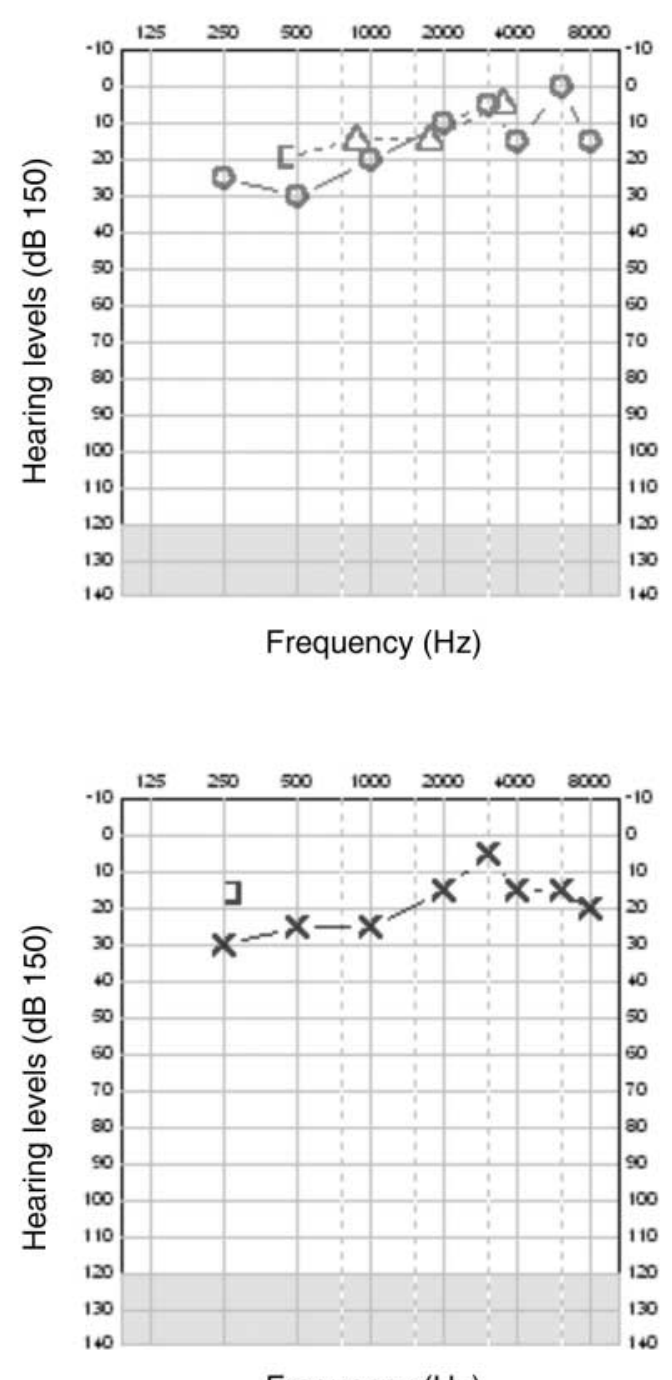

Frequency $(\mathrm{Hz})$

Figure 4 Pure tone audiogram. $\mathrm{O}$, right masked air conduction; $\mathrm{X}$, left masked air conduction; $\triangle$, unmasked bone conduction; $\mathbf{C}$, masked right bone conduction; I, left masked bone conduction octave, from $0.5-8 \mathrm{kHz}$. More sensitized audiometric measures, audioscan and Békésy audiometry sweep through the frequencies. ${ }^{5}$ The audioscan, for example, performs a frequency sweep of up to 64 frequencies within an octave, from $0.125-16 \mathrm{kHz}$, providing more detailed information about the hearing thresholds. Where there is a suspicion of progressive or genetic hearing impairment, regular audiometric assessment, especially using sensitized audiometric testing, can be helpful. In cases of suspected genetic hearing impairment, tests on first degree relatives can detect similar patterns in audiometric configurations, as illustrated in Case 3. Progressive hearing loss can be associated with a number of cochlear disorders, including a wide vestibular aqueduct, auto-immune ear disease and endolymphatic disorders. Menière's disorder is but one expression of a family of disorders which have endolymphatic hydrops as the underlying pathology. ${ }^{6}$ Symptomatic endolymphatic hydrops can present with aural fullness, episodic vertigo, tinnitus and fluctuating hearing loss. The presence of the different symptoms varies as does the underlying aetiology while the symptoms can also be of varying degrees. Note should be taken of associated aural or neurological symptoms. Electrophysiological tests, e.g. otoacoustic emissions, auditory brainstem responses, etc., may be useful where there is a perceived unilateral hearing difficulty, as they may detect a functional deficit along the auditory pathway from the cochlea to the brainstem.

Neurological disorders, e.g. multiple sclerosis, cerebrovascular events, etc., may affect the central auditory pathways despite a normal peripheral hearing system. They can, therefore, affect the perception of sounds without affecting either the auditory thresholds or speech recognition in a quiet setting. It may be helpful to look at the central auditory pathway to assess interhemispheric transfer of auditory-linguistic information. Neoplastic causes of hearing difficulty are important to identify as early Right Ear

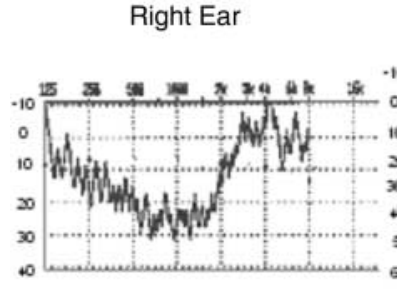

Left Ear

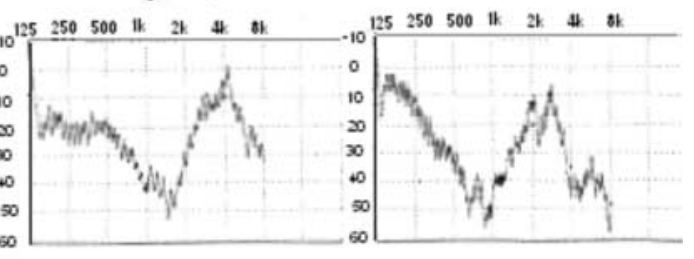

Left Ear

Left Ear

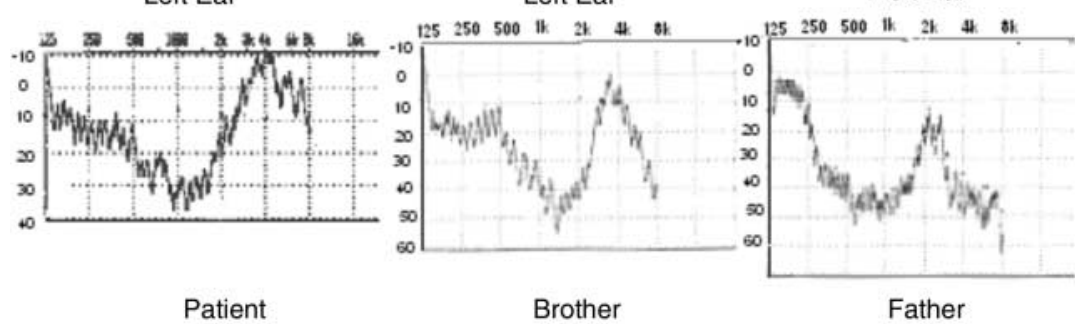

Patient

Brother 
management may significantly reduce morbidity. Although vestibular Schwannomas represent about $8 \%$ of all intracranial tumours, they constitute 75\%-90\% of cerebello-pontine angle lesions. ${ }^{7}$ Other cranial nerve neuromas make up less than $1 \%-2 \%$ of those lesions. Typically, cerebello-pontine angle lesions, including vestibular Schwannomas, present with unilateral symptoms of hearing loss or tinnitus-less commonly, imbalance. Hearing is normal, however, in $5 \%$ of patients with a vestibular Schwannoma.

As with many medical problems taking an appropriate history is invaluable, both to decide what tests are necessary and also to reach a diagnosis. Once a detailed history is taken into account, a normal 'hearing test', i.e. pure tone audiometry, may be just the beginning. The history may suggest the need for further tests of hearing or monitoring of hearing thresholds. The particular features that should prompt further investigation and monitoring are progressive symptoms, unilateral symptoms, associated audio-vestibular symptoms, associated neurological symptoms or a family history of hearing difficulties. With the current increasing pressure on outpatient services due to government targets, it may be appropriate to allow open appointments where, after appropriate counselling/hearing therapy, it is left to the patient to contact the department if further problems arise rather than discharging the patient.
While hearing difficulties can exist with a normal basic hearing test, abnormalities may be found on closer inspection, or, symptoms may evolve so that the diagnosis is apparent over time. This collection of cases illustrates that a normal hearing test does not always reflect a normal hearing system but, rather, may just be the beginning of the investigation process.

Competing interests None declared.

\section{REFERENCES}

1 Zhao F, Stephens D. Subcategories of patients with King-Kopetzky syndrome. Br J Audiol 2000;34:241-56

2 Saunders G, Haggard MP. The clinical assessment of obscure auditory dysfunction - auditory and psychological factors. Ear Hear 1989;10: 200-8

3 Higson J, Haggard M, Field D. Validation of parameters for assessing obscure auditory dysfunction - robustness of determinants of OAD status across samples and test methods. Br J Audiol 1994;28:27-39

4 Stephens D, Zhao F, Kennedy V. Is there an association between noise exposure and King Kopetzky Syndrome? Noise Health 2003;5:55-62

5 Laroche C, Hetu R. A study of the reliability of automatic audiometry by the frequency scanning method (audioscan). Audiol 1997;36:1-18

6 Schuknecht, HF, Gulya, AJ. Endolymphatic hydrops. An overview and classification. Ann Otol Rhinol Laryngol 1983;106(sSuppl):1-20

7 Brakcmann DE, Arriaga MA. Extra-axial neoplasms of the posterior fossa. In: Cummings CW, Fredrickson JM, Krause CJ, Harker LA, Schuller DE, eds. Otolaryngology Head and Neck Surgery, 3rd edn. St Louis: Mosby, 1998:3294-15 